\title{
Cyclin-Dependent Kinase Activity Is Required for Apoptotic Death But Not Inclusion Formation in Cortical Neurons after Proteasomal Inhibition
}

\author{
Hardy J. Rideout, ${ }^{1}$ Qiaohong Wang, ${ }^{1}$ David S. Park, ${ }^{3}$ and Leonidas Stefanis ${ }^{1,2}$ \\ Departments of ${ }^{1}$ Neurology and ${ }^{2}$ Pathology, Columbia University, New York, New York 10032, and ${ }^{3}$ Neuroscience Research Institute, University of Ottawa, \\ Ottawa, ON K1H 8M5 Canada
}

\begin{abstract}
Growing evidence suggests that the proteasome may be dysfunctional in a number of neurodegenerative disorders, including Lewy body diseases. We have reported previously that application of pharmacological inhibitors of the proteasome to cultured cortical neurons leads to apoptotic death and formation of ubiquitinated cytoplasmic inclusions. A number of cell cycle regulatory proteins are known to be degraded by the proteasome. In light of the emerging role of aberrant cell-cycle activation in neuronal cell death, we have assessed the involvement of cell-cycle components in the effects induced by proteasomal inhibitors in cortical neurons. Death and mitochondrial dysfunction induced by lactacystin and other pharmacological inhibitors of the proteasome were prevented by flavopiridol, a specific inhibitor of cyclin-dependent kinases (Cdks). Molecular expression of the Cdk inhibitors p16 or p27, or of dominant-negative Cdk2, Cdk4, or Cdk6 was also protective against lactacystin-induced death. Flavopiridol blocked the induction of retinoblastoma protein $(\mathrm{pRb})$ phosphorylation that occurred after lactacystin application, and expression of a mutant $\mathrm{pRb}$ that lacked phosphorylation sites was neuroprotective. These results suggest that in cortical neurons, proteasomal inhibition leads to a cell death pathway that is dependent on Cdk activation and pRb inactivation. Although cyclins D1 and E were sequestered within the ubiquitinated inclusions formed at late time points after lactacystin application, the formation of ubiquitinated inclusions was unaffected by Cdk inhibition. This suggests that there are parallel pathways regulating neuronal death and inclusion formation elicited by proteasomal inhibition in cortical neurons.
\end{abstract}

Key words: cell cycle; retinoblastoma; proteasome; apoptosis; flavopiridol; ubiquitin

\section{Introduction}

Recent evidence suggests that dysfunction of the ubiquitindependent proteolytic system may play a role in a number of neurodegenerative conditions. In particular, dysfunction of the proteasome, the proteolytic complex involved in the degradation of polyubiquitinated proteins, has been implicated as a contributing factor in Parkinson's disease (PD), Alzheimer's disease (AD), and Huntington's disease (HD) (Lam et al., 2000; McNaught et al., 2001; Waelter et al., 2001). The evidence supporting a role for dysfunction of the ubiquitin-proteasomedependent proteolytic pathway is particularly strong for Lewy body (LB) diseases (Chung et al., 2001; McNaught et al., 2001). Both genetic (Leroy et al., 1998; Shimura et al., 2000) and biochemical (Iwatsubo et al., 1996; McNaught and Jenner, 2001; Stefanis et al., 2001; Tanaka et al., 2001) data suggest that such a dysfunction could participate in LB disease pathogenesis. We have found that application of pharmacological proteasome inhibitors to neuronal PC12 cells (Rideout et al., 2001a) or cortical neurons (Rideout and Stefanis, 2002) induces cell death and

Received 0ct. 22, 2002; revised 0ct. 22, 2002; accepted Nov. 25, 2002.

This work was supported by the American Parkinson Disease Foundation (H.J.R., L.S.), the Matheson Foundation the Parkinson's Disease Foundation (L.S.), and the Canadian Institutes of Health Research (CIHR) (D.S.P.). L.S. is the recipient of a Career Award in Biomedical Sciences from the Burroughs Wellcome Foundation. D.S.P. is a CIHR Scholar and a Glaxo Wellcome Chair Recipient. We thank Manish Noticewalla and Andrea Perger for blinded counts of apoptotic neurons.

Correspondence should be addressed to Hardy J. Rideout, Department of Neurology, Columbia University, Black Building 326, 650 West 168th Street, New York, NY 10032. E-mail: HR227@columbia.edu.

Copyright $\odot 2003$ Society for Neuroscience $\quad 0270-6474 / 03 / 231237-09 \$ 15.00 / 0$ ubiquitin/ $\alpha$-synuclein-positive fibrillar cytoplasmic inclusions, thus mimicking the hallmarks of LB diseases, and others have shown that proteasome inhibitors induce apoptotic death of postmitotic neurons accompanied by caspase activation and mitochondrial dysfunction (Qiu et al., 2000).

In view of the potential importance of proteasomal inhibition in LB disease pathogenesis, we have investigated further the molecular pathways induced by pharmacological proteasomal inhibition in primary cortical neurons. In the current work, we have focused our attention on activation of elements of the cell cycle. Studies in cycling cells have shown that a number of proteins that participate in cell-cycle regulation are degraded by the proteasome. Of the molecules that function at the $\mathrm{G}_{1} / \mathrm{S}$ phase of the cell cycle, cyclin D1, cyclin E, and the cyclin-dependent kinase inhibitor (Cdki) p27 are thought to be degraded by the proteasome (Pagano et al., 1995; Pagano, 1997; King et al., 1998). In cycling cells, application of specific proteasomal inhibitors leads to cellcycle arrest (Fenteany et al., 1995; Pagano et al., 1995) and, depending on the cell type, the time of exposure, and the dosage of the inhibitor, to cellular differentiation (Obin et al., 1999; Baldassarre et al., 2000) or to cell death (Drexler, 1997).

Although there is evidence for aberrant cell-cycle activation in AD (Busser et al., 1998; Nagy et al., 1998), very little is known about such deregulation in LB diseases. A recent study reported alterations in the mRNA levels of cell-cycle components after 1-methyl-4-phenyl-1,2,3,6-tetrahydropyridine administration in mice, suggesting that in this animal model of Parkinsonism cell 
cycle deregulation may play a role in neuronal cell death (Grunblatt et al., 2001).

To investigate the effects of proteasomal inhibition on cellcycle regulation in cortical neurons, we have assessed regulatory elements involved in the $G_{1} / S$ transition. We find that proteasomal inhibition in this system induces activation of cell-cycle elements at the $G_{1} / S$ interphase, and that such activation is essential for proteasome inhibition-induced apoptosis but is not necessary for cytoplasmic inclusion formation.

\section{Materials and Methods}

Cortical neuronal cultures. Cultures of rat embryonic day 18 (E18) cortical neurons were prepared as described previously (Stefanis et al., 1999; Rideout et al., 2001b). Cortices from E18 rat fetuses were removed aseptically into sterile PBS, cleaned free of meningeal tissue, minced, and mechanically dissociated using a flame-polished Pasteur pipette. Dissociated cells were plated onto poly-D-lysine-coated 96 well, 24 well, or 35 $\mathrm{mm}$ plastic dishes for survival assays and protein chemistry, or onto glass coverslips for immunocytochemistry at a density of $\sim 150,000-200,000$ per $\mathrm{cm}^{2}$. Cells were maintained in neurobasal medium (Invitrogen, Carlsbad, CA) with B27 serum-free supplements, L-glutamine (0.5 mM), and penicillin-streptomycin.

Constructs and viral infection. cDNAs encoding the Cdkis p16, p27, or dominant-negative Cdk4 (DN Cdk4), Cdk6 (DN Cdk6), Cdk2 (DN Cdk2), and Cdk3 (DN Cdk3), were subcloned into the Sindbis viral expression vector as described previously (Park et al., 1997, 1998). Each insert also contained a Flag tag. A control for each construct (except for DN Cdk2), containing a premature stop codon or a missing initiation codon, was also generated that did not possess a Flag tag. The plasmids were linearized using $P v u \mathrm{I}$, ethanol precipitated, and in vitro transcribed using an RNA capping kit (Stratagene, La Jolla, CA). The RNA was transfected into baby hamster kidney (BHK) cells, and the supernatant containing viral particles was collected after $24 \mathrm{hr}$ and stored in aliquots at $80^{\circ} \mathrm{C}$. The viral titer (plaque-forming units per milliliter) was determined in BHK cells infected with serial dilutions of viral stock. Cortical neurons were infected $24 \mathrm{hr}$ after plating at a multiplicity of infection (MOI) of $1-2$, as described previously (Park et al., 1997, 1998). After infection, the neurons were cultured for an additional $24 \mathrm{hr}$ before treatment with proteasome inhibitors or assessment of protein expression by Western immunoblot as described below.

Recombinant adenoviruses encoding mutant $\mathrm{pRb}(\Delta \mathrm{K} 11 \mathrm{Rb})$ or enhanced green fluorescent protein (EGFP) as a control were generated as described previously (Park et al., 2000). The $\Delta \mathrm{K} 11 \mathrm{Rb}$ cDNA was a kind gift from Dr. Eldad Zacksenhaus (University of Toronto, Toronto, Canada). It is based on mouse $\mathrm{pRb}$ and has the following substitutions at phosphorylation sites: T246A, T350R, S601A, S605A, S773A, S781A, S788A, S800A, S804E, T814A, and T819A (Brown et al., 1999). Neurons were infected as described previously (Park et al., 2000) on days 1-3 after plating at an MOI of 150 for a period of $1 \mathrm{hr}$ and then cultured for an additional $24-36 \mathrm{hr}$ before addition of proteasomal inhibitors.

Induction of cell death by proteasomal inhibition. On days 2-3 in vitro, the highly specific inhibitors of the $26 \mathrm{~S}$ proteasome lactacystin, PSI, or epoxomicin (Figueiredo-Pereira et al., 1994; Fenteany et al., 1995; Fenteany and Schreiber, 1998; Meng et al., 1999) were added to the cultures. Lactacystin (Kamiya Biomedical, Thousand Oaks, CA) was prepared in sterile $\mathrm{dH}_{2} \mathrm{O}$ at a stock concentration of $1 \mathrm{~mm}$. PSI and epoxomicin (Calbiochem, La Jolla, CA) were prepared in DMSO at stock concentrations of 10 and $1 \mathrm{~mm}$, respectively. At indicated times after addition of proteasomal inhibitors, cells were lysed and neuronal viability was estimated using counts of intact nuclei as described previously (Stefanis et al., 1999; Rideout and Stefanis, 2002). Total surviving neurons are expressed as the percentage of untreated cultures at the time of cell lysis. In parallel experiments, the percentage of apoptotic nuclei, as an index of neuronal cell death, was assessed using the nuclear dye Hoechst 33342 (1 $\mu \mathrm{g} / \mathrm{ml}$; Sigma, St. Louis, MO), as described previously (Stefanis et al., 1999; Rideout and Stefanis, 2002). For the purposes of quantifying apoptotic neuronal death, only nuclei with two or more condensed apoptotic bodies were scored as positive. Other cultures were exposed to lactacystin in the presence of the general caspase inhibitors Boc-aspartyl (OME)fluoromethylketone (BAF) (100 $\mu \mathrm{M}$; Enzyme Systems Products, Livermore, CA), the transcriptional inhibitor actinomycin D (10 $\mu \mathrm{M}$; Sigma), or the Cdki flavopiridol ( $1 \mu \mathrm{m}$; generously provided by Dr. P. Worland, National Institutes of Health, Bethesda, MD).

Immunofluorescence. Neurons grown on glass coverslips were fixed in freshly prepared $3.7 \%$ formaldehyde for $25 \mathrm{~min}$ at $4^{\circ} \mathrm{C}$ and then incubated with $10 \%$ normal goat serum with $0.4 \%$ Triton X-100 to block nonspecific binding, followed by incubation with the primary antibody for $1 \mathrm{hr}$ at room temperature. Specific antibodies used were rabbit antiubiquitin (1:100; Dako, Glostrup, Denmark), mouse anti-cyclin D1 (1: 20; Santa Cruz Biotechnology, Santa Cruz, CA), rabbit anti-cyclin E (1: 100; Santa Cruz Biotechnology), mouse anti-p27 (1:100; Santa Cruz Biotechnology), mouse anti-cytochrome $c$ (1:500; PharMingen, San Diego, CA), mouse anti-GFP (1:100; Santa Cruz Biotechnology), mouse anti-pRb (1:500; PharMingen), or mouse anti-extracellular signalregulated kinase-2 (ERK2) (1:300; Santa Cruz Biotechnology). After incubation with fluorescent secondary antibodies (Cy2, 1:100; or Cy3, 1:250; Jackson ImmunoResearch, West Grove, PA), coverslips were placed on glass slides and visualized using standard epifluorescence or confocal microscopy (LSM410; Ziess, Thornwood, NY).

We have reported previously that proteasomal inhibition leads to formation of ubiquitinated cytoplasmic inclusions in PC12 cells and cortical neurons (Rideout et al., 2001a; Rideout and Stefanis, 2002). To quantify such inclusions, we assessed the percentage of cells in the cultures that contained a discrete area of increased ubiquitin immunoreactivity not encompassing the entire cytoplasmic volume. Counts were performed as above for nuclear accumulation of the cyclins. To quantify neurons that have lost mitochondrial cytochrome $c$, neurons were evaluated for the presence of punctate cytoplasmic cytochrome $c$ immunoreactivity. Those neurons that had completely lost cytochrome $c$ staining or those in which cytochrome $c$ had assumed a diffuse cytoplasmic pattern were counted as negative. Triplicate coverslips were assessed, counting at least 100 neurons each by a rater blinded to the experimental conditions.

Subcellular fractions. To identify changes in subcellular localization of specific proteins, enriched mitochondrial fractions were obtained using differential centrifugation. Briefly, cells were washed with ice-cold PBS and centrifuged. The pellets were resuspended in $200 \mu \mathrm{l}$ of ice-cold buffer A (300 mm sucrose, 1 mM EGTA, 20 mM morpholinopropanesulfonate, and a protease inhibitor mixture; Complete, Roche Products, Hertforshire, UK) and incubated on ice for $20 \mathrm{~min}$. The cell suspension was transferred to a $2 \mathrm{ml}$ glass homogenizer and disrupted with 40 strokes. The nuclei and any remaining unbroken cells were pelleted by centrifugation at $1000 \mathrm{rpm}$ for $10 \mathrm{~min}$ at $4^{\circ} \mathrm{C}$. The supernatant was centrifuged again at $14,000 \mathrm{rpm}$ for $15 \mathrm{~min}$ at $4^{\circ} \mathrm{C}$ to obtain an enriched mitochondrial pellet, which was resuspended in $50 \mu \mathrm{l}$ of lysis buffer $(25 \mathrm{~mm}$ HEPES, pH 7.4, 5 mm EDTA, 1 mm EGTA, $5 \mathrm{~mm} \mathrm{MgCl}_{2}$, and a protease inhibitor mixture) containing $1 \%$ Triton $\mathrm{X}-100$. This enriched mitochondrial lysate was used for Western immunoblotting as described below.

Western immunoblotting. For total cell lysates, cortical neurons were rinsed in ice-cold PBS, removed from the culture dish by scraping or trituration, and then solubilized in SDS sample buffer containing 5\% $\beta$-mercaptoethanol to generate total cell lysates. Proteins were separated by SDS-PAGE (12\%), transferred to nitrocellulose membranes, and incubated with primary antibodies. Specific antibodies used were: antiphosphoRB (1:1000; New England Biolabs, Beverly, MA), native pRb (1:1000; PharMingen), anti-GFP (1:300; Santa Cruz Biotechnology), anti-Flag (1:400; Sigma), and p27, cyclin D1, and cyclin E (all 1:300; Santa Cruz Biotechnology). Protein bands were visualized with horseradish peroxidase-conjugated secondary antibodies (Pierce, Rockford, IL) and enhanced chemiluminescence (Pierce). To control for protein loading, the membranes were stripped and reprobed with rabbit anti-ERK (1: 5000; Santa Cruz Biotechnology). Mitochondria-enriched fractions were similarly separated by SDS-PAGE (12\%), and immunoblotting was performed with a mouse anti-cytochrome $c$ antibody (1:2000, clone 7H8.2C12; PharMingen). As a control for mitochondrial content and protein loading, membranes were stripped and reprobed with mouse 


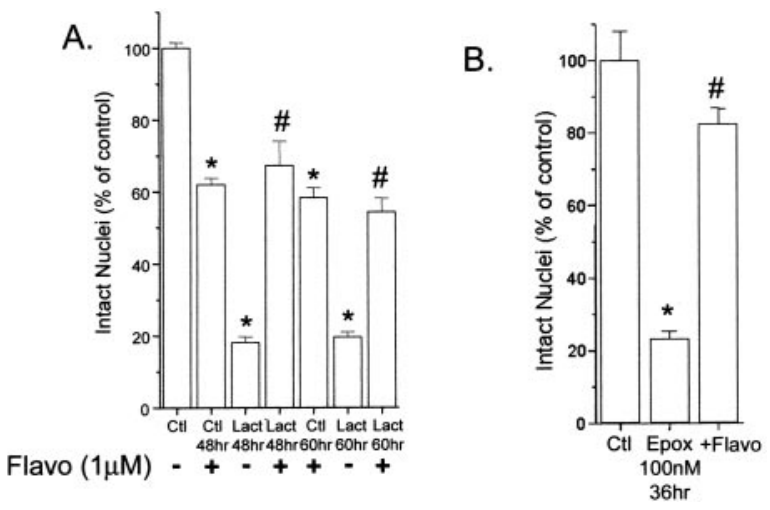

Figure 1. Cdk inhibition prevents proteasomal inhibition-induced neuronal death. A, Cortical neurons were treated with lactacystin $(L a c t ; 10 \mu \mathrm{M})$ in the presence or absence of flavopiridol (Flavo; $1 \mu \mathrm{M}$ ) and lysed; intact nuclei were then counted on a hemocytometer. Bars represent the mean number of intact nuclei expressed as a percentage of untreated control $(C t /)$ cultures from four replicate wells. ${ }^{*} p<0.001$ compared with control; ${ }^{\#} p<0.001$ compared with lactacystin alone. Similar results were achieved in four additional experiments. $B$, Cultured cortical neurons treated with epoxomicin (Epox; $100 \mathrm{~nm}$ ) alone or simultaneously with flavopiridol $(1 \mu \mathrm{m})$ for a period of $36 \mathrm{hr}$ were lysed, and the intact nuclei were counted on a hemocytometer. Bars represent the mean number of intact nuclei expressed as a percentage of untreated control cultures from four replicate wells. ${ }^{*} p<0.001$ compared with control; ${ }^{\#} p<$ 0.001 compared with lactacystin alone.

anti-cytochrome $c$ oxidase subunit IV (COXIV; 1:2500; Molecular Probes, Eugene, OR).

Statistical analyses. Comparisons between groups were made by ANOVA with Newman-Keuls post hoc comparisons or with Student's $t$ test unless otherwise noted. The level of significance was set at 0.05 .

\section{Results}

The Cdki flavopiridol prevents proteasomal inhibitioninduced apoptotic death by acting upstream of the mitochondrial checkpoint

We and others (Qiu et al., 2000; Rideout and Stefanis, 2002) have shown that pharmacological inhibition of the proteasome induces apoptotic death of cultured embryonic cortical neurons. To determine whether activation of Cdks is involved in proteasomal inhibition-induced cortical neuron death, we applied the specific proteasomal inhibitor lactacystin $(10 \mu \mathrm{M})$, alone or together with the specific pharmacological inhibitor of Cdks, flavopiridol $(1 \mu \mathrm{M})$. Flavopiridol prevented lactacystin-induced death of cortical neurons as determined by counts of intact nuclei (Fig. 1A). Despite some toxicity induced by flavopiridol alone with prolonged treatment, there was substantial protection, even up to $60 \mathrm{hr}$ after lactacystin application (Fig. $1 \mathrm{~A}$ ). Similar protection was seen against neuronal death induced by two other specific proteasomal inhibitors, epoxomicin (Fig. $1 B$ ) or PSI (data not shown).

To examine the site of action of flavopiridol within the neuronal apoptotic pathway induced by proteasomal inhibition, we focused first on events occurring at the level of the mitochondria. In many situations, the release of cytochrome $c$ from mitochondria is a pivotal event that leads to caspase activation and subsequent apoptosis. Qiu et al. (2000) have previously shown such release of cytochrome $c$ in proteasome inhibitor-treated cortical neurons. We first assessed the release of cytochrome $c$ from mitochondria of lactacystin-treated neurons by immunofluorescence. In control neurons, cytochrome $c$ was localized to the mitochondria, and colocalized with the mitochondrial dye chloromethyltetramethyrosamine (Molecular Probes) (Fig. $2 \mathrm{~A}$, data
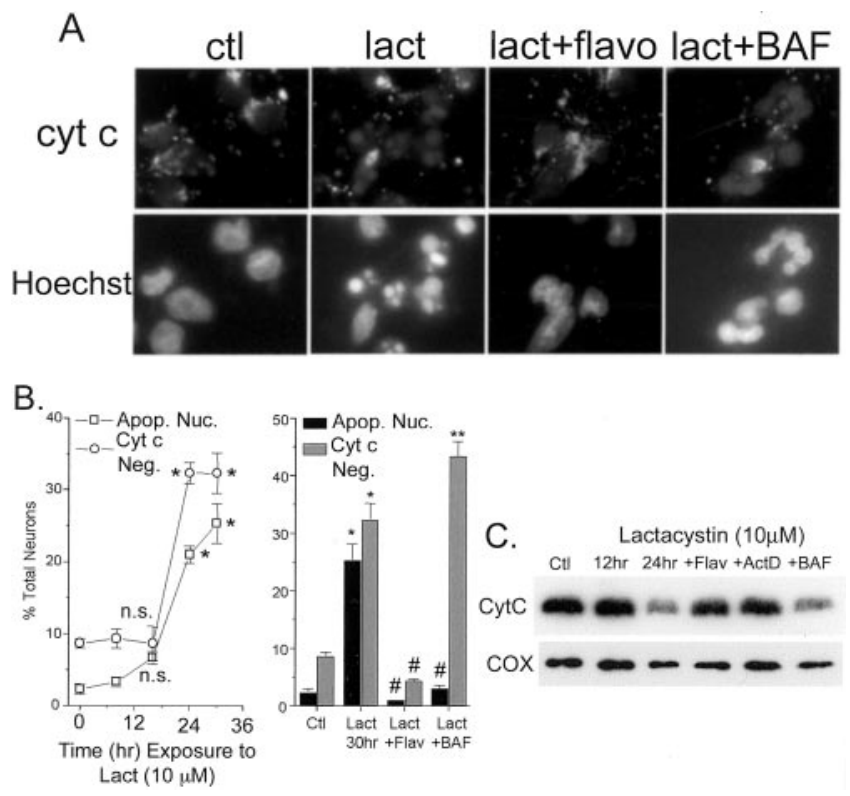

Figure 2. Flavopiridol acts upstream of mitochondrial dysfunction in proteasome inhibitortreated cortical neurons. A, Cortical neurons were treated with lactacystin (lact; $10 \mu \mathrm{m}$ ) in the presence or absence of flavopiridol ( flavo; $1 \mu \mathrm{m}$ ) or BAF (100 nM) for the indicated times, fixed, and immunostained for anti-cytochrome $c$ (cyt c) plus the nuclear dye Hoechst. ctl, Control. $B$, Neurons were treated and immunostained as in $A$, and the percentage of neurons that had lost punctate mitochondrial cytochrome c (Cyt c) staining (open circles/gray bars) or that had apoptotic nuclei (Apop. Nuc.; open squares/black bars) was determined. The data points are representative of two independent experiments. n.s., Not significant. Lact, lactacystin; Flav, flavopiridol. ${ }^{*} p<0.001$ compared with control; ${ }^{* *} p<0.01$ compared with lactacystin alone; ${ }^{\#} p<$ 0.001 compared with lactacystin alone. $C$, Neurons were treated as indicated and were separated into heavy membrane fractions enriched in mitochondria. Proteins were separated by SDS-PAGE (12\%), blotted to nitrocellulose membranes, and probed with mouse anticytochrome $c(1: 2000)$. The membranes were then stripped and reprobed with mouse antiCOXIV (1:2500). ActD, Actinomycin D.

not shown). This localization did not change for $16 \mathrm{hr}$ after lactacystin treatment. Starting at the time point between 16 and 24 hr after lactacystin exposure, there was a loss of punctate mitochondrial cytochrome $c$ immunoreactivity that occurred in both apoptotic and nonapoptotic neurons (Fig. $2 A, B$ ). Although both flavopiridol and the general caspase inhibitor BAF prevented apoptosis, only flavopiridol, but not BAF, inhibited cytochrome $c$ release (Fig. $2 A, B$ ). These results indicate that $\mathrm{Cdk}$ activation occurs upstream of cytochrome $c$ release in lactacystin-induced apoptosis of cortical neurons, whereas caspase activation is situated downstream of this release.

To verify these results, we also used Western immunoblotting of crude mitochondrial fractions of cortical neurons. Lactacystin induced a time-dependent decrease in cytochrome $c$ content in mitochondria-enriched heavy membrane fractions. Simultaneous treatment with flavopiridol, but not BAF, prevented this release, confirming the immunofluorescence results (Fig. 2C). We have found previously that treatment with the transcriptional inhibitor actinomycin D prevented proteasomal inhibitioninduced apoptosis of cortical neurons (Rideout and Stefanis, 2002). Actinomycin D, like flavopiridol, prevented the release of cytochrome $c$ from mitochondria (Fig. 2C), consistent with results in other paradigms (Deshmukh and Johnson, 1998; Neame et al., 1998). We were not able to consistently see an accumulation of cytochrome $c$ in the cytoplasmic fraction after lactacystin application, presumably because of its rapid degradation (Desh- 
mukh and Johnson, 1998; Neame et al., 1998; Stefanis et al., 1999). As reported by Qiu et al. (2000), proteasomal inhibition also led to a decrease in mitochondrial membrane potential $\left(\Delta \Psi_{\mathrm{M}}\right)$ as estimated by accumulation of the fluorescent dye tetramethylrosamine (Molecular Probes). As with cytochrome $c$ release, this loss was inhibited by flavopiridol and actinomycin D, but not BAF (data not shown).

We conclude that the release of cytochrome $c$ from the mitochondria and the loss of $\Delta \Psi_{\mathrm{M}}$ require Cdk activity and new or ongoing mRNA synthesis but not caspase activation. In conjunction, these results indicate that the Cdki flavopiridol and the transcriptional inhibitor actinomycin D prevent proteasomal inhibition-induced neuronal apoptotic death by acting at a point upstream of the mitochondrial checkpoint.

\section{Lactacystin induces Cdk-dependent phosphorylation of retinoblastoma protein}

Based on the survival-promoting effects of the Cdki flavopiridol in this model, we anticipated that proteasomal inhibition would lead to Cdk activation, and that this activation would be blocked by flavopiridol. Furthermore, based on the fact that flavopiridol inhibited the release of cytochrome $c$ from mitochondria, we anticipated that such Cdk activation would occur at an early point in this cell death pathway, before mitochondrial changes and the resultant caspase activation. To examine these possibilities, we assessed the phosphorylation of $\mathrm{pRb}$, which is a major event that occurs after Cdk activation at the $\mathrm{G}_{1} / \mathrm{S}$ phase of the cell cycle (Harbour et al., 1999; Harbour and Dean, 2000). Phosphorylation of $\mathrm{pRb}$ is detected at early stages in a number of models of neuronal apoptotic death in which cell cycle elements are involved (Giovanni et al., 1999; Padmanabhan et al., 1999; Park et al., 2000). We treated cortical neurons with lactacystin for various times and probed total cellular lysates with an antibody that specifically recognizes pRb phosphorylated at Ser795. Proteasomal inhibition with lactacystin led to an early increase in the amount of phosphorylated $\mathrm{pRb}$ in cortical neurons at $4-8 \mathrm{hr}$ after exposure (Fig. 3A). As anticipated, flavopiridol prevented the increase in $\mathrm{pRb}$ phosphorylation (Fig. $3 B-D$ ). There was no induction of native $\mathrm{pRb}$ with lactacystin treatment (Fig. 3B, middle panel), indicating that lactacystin induces a specific post-translational modification of $\mathrm{pRb}$ rather than simply an upregulation of protein levels. The transcriptional inhibitor actinomycin D had no effect on the increase in $\mathrm{pRb}$ phosphorylation (Fig. $3 C, D$ ), indicating that transcriptional regulation is not involved in the induction of Cdk activation and resultant $\mathrm{pRb}$ phosphorylation, and situating the site of action of actinomycin $\mathrm{D}$ at a later point in this apoptotic pathway.

We conclude that $\mathrm{pRb}$ is phosphorylated at early time points (4-8 hr) after lactacystin application to cultured cortical neurons, and that this phosphorylation is dependent on Cdk but not on transcription.

\section{Molecular inhibitors of Cdk4, Cdk6, and Cdk2 attenuate lactacystin-induced cell death}

Flavopiridol acts as a specific inhibitor of multiple Cdks (Losiewicz et al., 1994). To verify that the protection achieved with this pharmacological approach was indeed caused by inhibition of Cdks, and to identify the specific Cdks involved, we infected the cultures with recombinant Sindbis viruses encoding molecular Cdkis. The Sindbis virus delivery system has been used to successfully express recombinant proteins in cultured primary neurons (Park et al., 1997, 1998). We first generated recombinant Sindbis viruses encoding the endogenous Cdkis p27 or p16, or

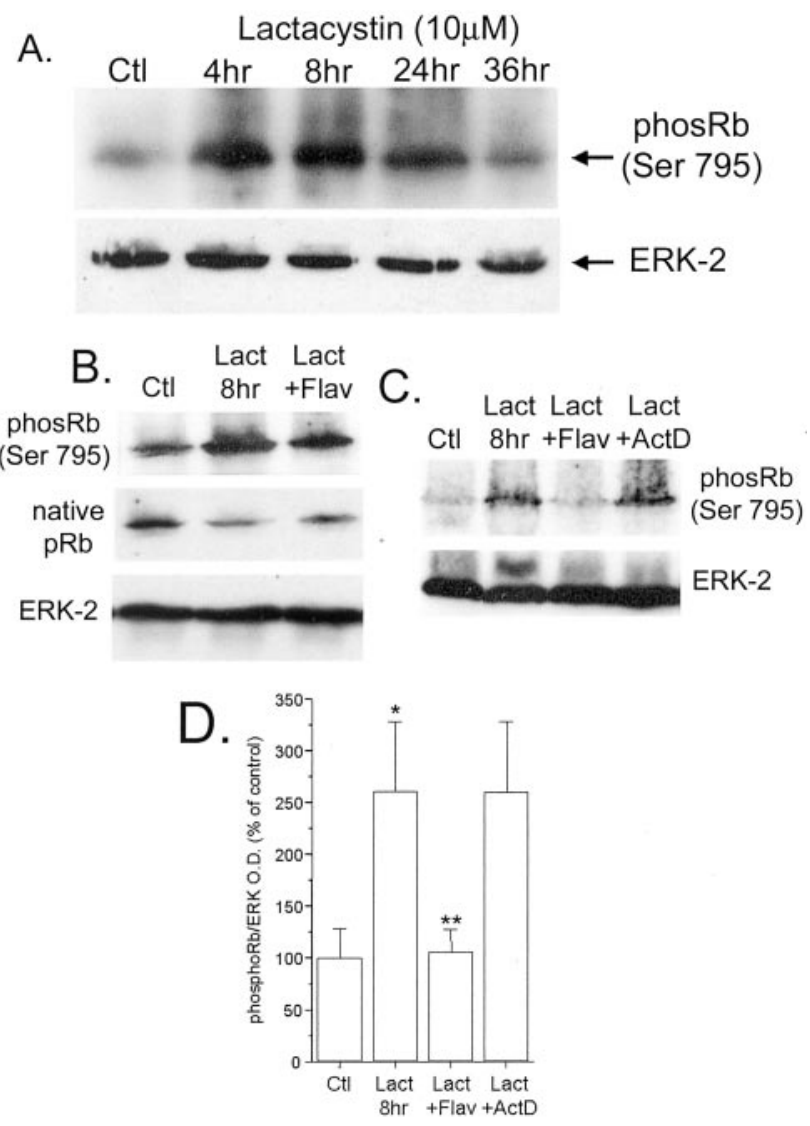

Figure 3. Retinoblastoma protein becomes phosphorylated early after proteasomal inhibition in cortical neurons. Cortical neurons were treated with lactacystin (10 $\mu \mathrm{m})$ and, at the indicated times, washed and solubilized in SDS sample buffer. The protein was separated by SDS-PAGE, blotted to nitrocellulose membranes, and probed with rabbit anti-phosphoRb (phosRb; Ser 795; 1:1000) or rabbit anti-native $\mathrm{pRb}(1: 1000, B)$. The membranes were stripped and reprobed with rabbit anti-ERK2 (1:5000, A, C. . , The optical density (0.D.) of bands of phosphoRb relative to ERK from four independent experiments was quantified and plotted. ${ }^{*} p<0.05$ compared with control; ${ }^{* *} p<0.05$ compared with lactacystin alone. Ctl, Control; Lact, Lactacystin; Flav, flavopiridol; ActD, Actinomycin D.

their respective controls, which had equal transcript lengths but were modified by the omission of an initiation codon or by the addition of a premature stop codon, so as to not express the proteins. Such controls are necessary, because Sindbis virus expression may have nonspecific effects, which vary according to the size of the recombinant transcripts (Park et al., 1997, 1998). It is thought that p27 preferentially inhibits Cdk2-associated activity (Hsieh et al., 2000), whereas p16 is more specific for Cdk4 and Cdk6 (Sandhu et al., 2000). Cortical neuron cultures were infected at an MOI of 1-2, and overexpression of recombinant proteins was verified by Western immunoblotting (Fig. 3A-C). Parallel cultures were treated with lactacystin $24 \mathrm{hr}$ after viral or sham infection. After an additional $36 \mathrm{hr}$ of lactacystin exposure, the neurons were lysed and the remaining intact nuclei counted. Because of the fact that the Sindbis virus induced some baseline toxicity in these cultures $(\sim 25-30 \%)$, for the purposes of quantifying survival, data from lactacystin-treated cultures are expressed relative to vehicle-treated cultures infected with the same viral construct. Expression of p27 and of p16, but not of their respective controls, significantly attenuated lactacystin-induced death (Fig. 4A).

To target specific Cdks, we expressed in a similar manner DN 

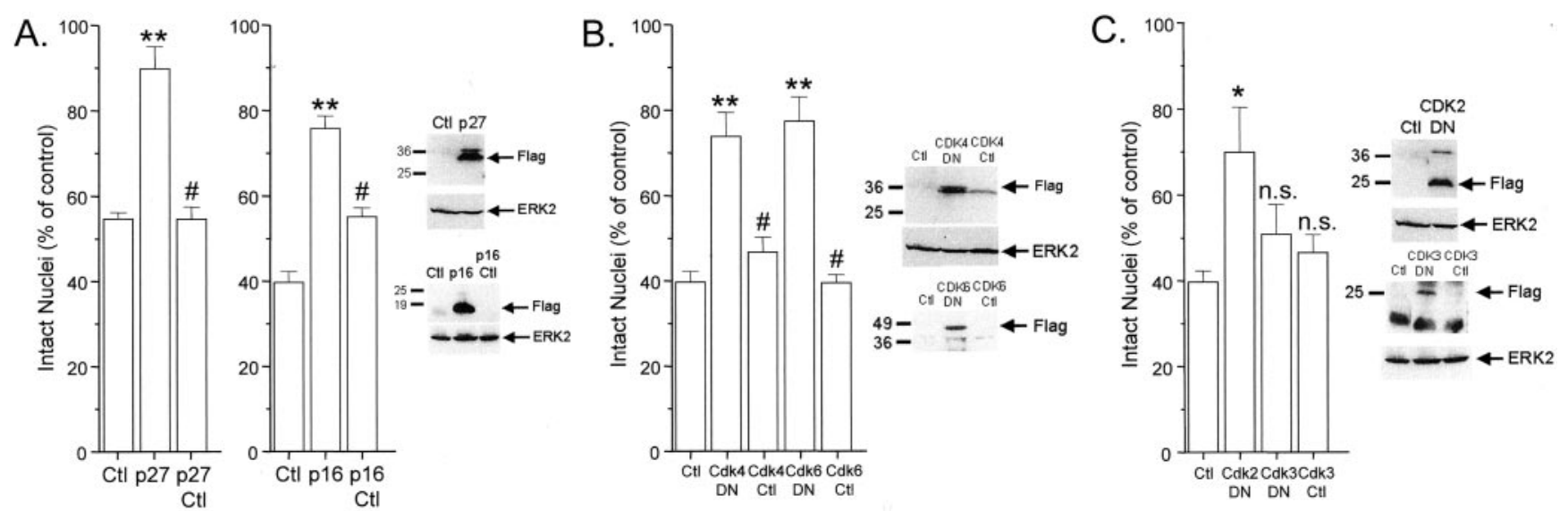

Figure 4. Cdk4-, Cdk6-, and Cdk2- but not Cdk3-associated activity is required for proteasomal inhibition-induced death of cortical neurons. Neurons were infected with Sindbis virus encoding Flag-tagged Cdkis p16 ( $A$ ) or p27, DN Cdk4 or Cdk6 (B), DN Cdk2 or Cdk3 ( $)$, or the appropriate control (Ctl) construct containing a premature stop codon. After $36 \mathrm{hr}$, total cellular lysates were prepared in SDS sample buffer and immunoblotted with mouse anti-Flag (1:400). The membranes were stripped and reprobed with rabbit anti-ERK2 (1:5000). 0ther neurons were treated $24 \mathrm{hr}$ after infection with lactacystin $(10 \mu \mathrm{m})$ and lysed $36 \mathrm{hr}$ later; intact nuclei were then counted. The mean number of intact nuclei was determined and expressed relative to untreated cultures infected with the same construct. ${ }^{* *} p<0.001$ compared with uninfected cultures; ${ }^{*} p<0.001$ compared with control constructs; ${ }^{*} p<0.05$ compared with control constructs. n.s., Not significantly different compared with uninfected cultures. All experiments were repeated two or three times, with similar results.

forms of specific Cdks, Cdk2, Cdk3, Cdk4, and Cdk6, in the cortical neuron cultures. We also infected the cultures with the respective control viruses in parallel. The DN Cdk3 control was also used as a control for DN Cdk2, because the transcripts were of equal length. Expression of the recombinant proteins was again verified by Western immunoblotting (Fig. 4B,C). Expression of DN Cdk4, DN Cdk6 (Fig. 4B), and DN Cdk2 (Fig. 4C), but not of DN Cdk3 (Fig. 4C), significantly attenuated lactacystin-induced death compared with controls.

We conclude that molecular inhibition of specific Cdks, and in particular of Cdk2, Cdk4, or Cdk6, results in improvement in survival, which was assessed in these experiments at $36 \mathrm{hr}$, at a time point at which considerable loss of neurons is detected. It is presumed that the site of action of such molecular inhibitors, like that of flavopiridol, is the Cdk activation that occurs at $4-8 \mathrm{hr}$ after lactacystin treatment.

\section{Expression of phosphorylation-deficient $\mathrm{pRb}$ protects neurons from lactacystin-induced apoptosis}

Previous work has shown that induced expression of a mutant form of $\mathrm{pRb}$ that is deficient in several phosphorylation sites protected cortical neurons against camptothecin-induced apoptotic death (Park et al., 2000). We expressed this mutant form of $\mathrm{pRb}(\mathrm{pRb} \Delta \mathrm{FK} 11)$ or a control protein, EGFP, using an adenoviral delivery system. As was done for the experiments with Cdkis or DN Cdks, we assessed the effects of mutant $\mathrm{pRb}$ on neuronal survival at time points at which significant apoptotic death is observed (24-30 hr) (Fig. 2 B). In this case, because the infection efficiency was less compared with that using the Sindbis virus, we immunostained the cultures and assessed only the neurons that expressed the proteins of interest. Counts of apoptotic nuclei in neurons that had been exposed to lactacystin $(10 \mu \mathrm{M})$ for 24 or 30 $\mathrm{hr}$ revealed significantly reduced apoptotic death in neurons expressing mutant $\mathrm{pRb}$ compared with those expressing EGFP (Fig. $5 A$ ), supporting a critical role for $\mathrm{pRb}$ phosphorylation inactivation in this model. Representative photomicrographs of lactacystin-treated neurons show an apoptotic nucleus of an EGFP-positive neuron and a nonapoptotic intact nucleus of a pRb-positive neuron (Fig. $5 B$ ). The protective effects of $\Delta \mathrm{FK} 11$ $\mathrm{pRb}$ were diminished in cells exposed to lactacystin for $\geq 36 \mathrm{hr}$
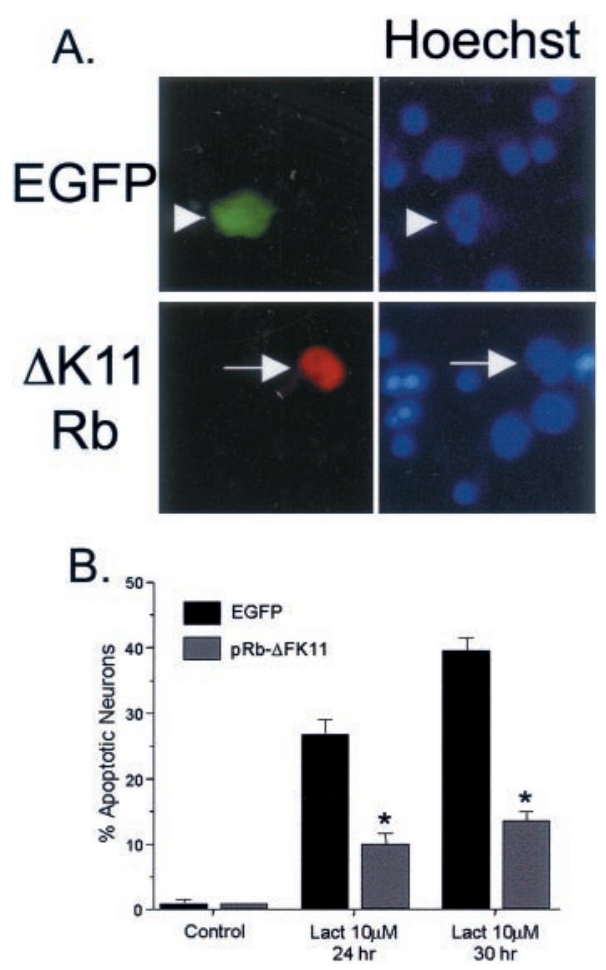

Figure 5. Expression of mutant $\Delta$ FK11-retinoblastoma protein is neuroprotective for lactacystin-treated cortical neurons. $A$, Neurons were infected with recombinant adenovirus encoding $\Delta$ FK11 Rb (arrow) or EGFP (arrowhead) for 24-36 hr, treated with lactacystin (10 $\mu \mathrm{M})$, fixed, and immunostained for anti-pRb or anti-EGFP and the nuclear dye Hoechst. $B$, Neurons infected as in $A$ were treated with vehicle or lactacystin (Lact; $10 \mu \mathrm{m}$ ) for $24-30 \mathrm{hr}$, fixed, and immunostained for anti-pRb or anti-EGFP and the nuclear dye Hoechst. The percentage of pRb- or EGFP-positive neurons with apoptotic nuclei was determined by a rater blinded to the experimental treatment. ${ }^{*} p<0.001$ compared with EGFP-expressing neurons treated with lactacystin.

(data not shown), consistent with the transient protective effect of this approach in the camptothecin model (Park et al., 2000).

We conclude that, as in the model of neuronal death induced by DNA damage, phosphorylation-inactivation of $\mathrm{pRb}$ is a crit- 


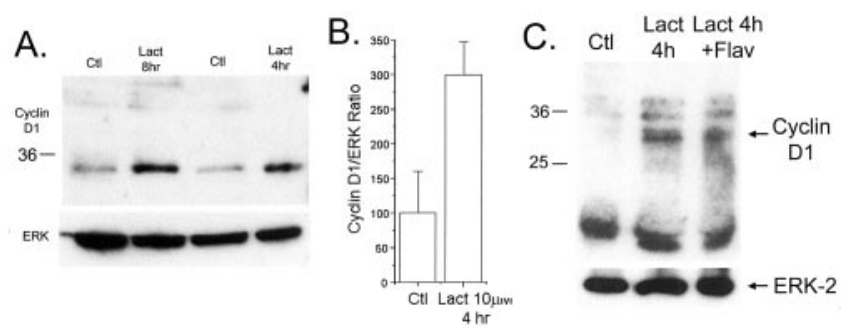

Figure 6. Cyclin D levels increase after inhibition of the proteasome and are not affected by Cdk inhibition. Cortical neurons were treated with lactacystin (Lact; $10 \mu \mathrm{M})$ for $4-8 \mathrm{hr}(A)$ or with lactacystin plus flavopiridol (Flav; $1 \mu \mathrm{m}$ ) for $4 \mathrm{hr}$ ( $($ ), washed, and solubilized in SDS sample buffer. The protein was separated by SDS-PAGE, blotted to nitrocellulose membranes, and probed with mouse anti-cyclin D1 (1:300). The membranes were stripped and reprobed with rabbit anti-ERK2 (1:5000) to demonstrate equal protein loading. C $t$, Control. The quantification in $B$ represents the ratio of cyclin D1 optical density to ERK2 optical density from five representative blots. ${ }^{*} p<0.05$ compared with control.

ical event in the apoptotic death elicited by inhibition of the proteasome.

\section{Cyclin D1 and cyclin E translocate to the nucleus after proteasomal inhibition}

In cycling cells, $\mathrm{pRb}$ phosphorylation occurs through the cooperative action of the cyclin D1/Cdk4/Cdk6 and the cyclin E/Cdk2 complexes (Lundberg and Weinberg, 1998; Harbour et al., 1999). Of these molecules, cyclin D1 and cyclin E are degraded by the proteasomal system in cycling cells, enabling an orderly progression through the cell cycle (Pagano, 1997; King et al., 1998). Little is known about the proteasomal degradation of cell-cycle components in noncycling, terminally differentiated cells such as neurons. Proteasomal inhibition would be expected to lead to an accumulation of cell-cycle proteins that are normally degraded by the ubiquitin-dependent proteolytic system. We therefore examined the levels of cyclins D1 and E, Cdk4 and Cdk6, and p27, in total cell lysates of cultures treated for 4-24 hr with lactacystin. These time points were selected to correspond to early points at which Cdk activation has been observed (4-8 hr), and later points when neuronal death begins to significantly increase (24 $\mathrm{hr}$ ). Western immunoblot analysis showed a significant increase in cyclin D1 levels at 4 and $8 \mathrm{hr}$ after exposure to lactacystin (Fig. $6 A, B$ ). The increase in levels was not affected by flavopiridol (Fig. $6 C)$. Western immunoblotting did not show any consistent changes in the levels of cyclin E, p27, Cdk4, or Cdk6 (data not shown).

We then examined the subcellular localization of these proteins by immunofluorescence. There was a significant increase in the percentage of neurons showing nuclear accumulation of cyclin D1 and cyclin E at 4 and $8 \mathrm{hr}$ after lactacystin application. This translocation was not blocked by flavopiridol, indicating that it was not dependent on Cdk activity. In contrast, by $24 \mathrm{hr}$ after lactacystin application, no nuclear cyclin D1 or cyclin E was seen (Fig. 7). By immunofluorescence, p27 localized constitutively within the nucleus, and the distribution did not change after lactacystin exposure (data not shown).

We conclude that cyclins D1 and E translocate in a Cdkindependent manner to the nuclei of cortical neurons shortly after lactacystin exposure. This translocation is evident at the same time points as pRb phosphorylation (Fig. 3). We attribute the fact that only a relatively small percentage of neurons show such translocation at the time points of 4 and $8 \mathrm{hr}$ to the asynchronous nature of responses that occur in individual cells within a population after exposure to the same stimulus. It is likely that
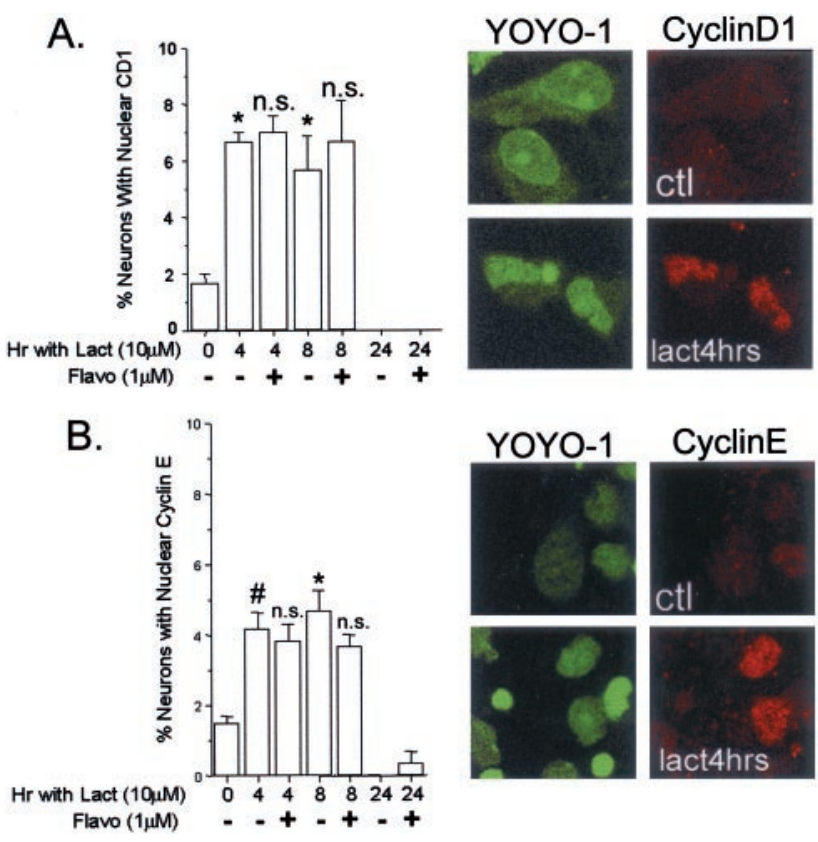

Figure 7. Cyclins D1 and Etransiently accumulate in the nucleus after proteasomal inhibition in cortical neurons. Neurons treated with lactacystin (Lact; $10 \mu \mathrm{m})$ with or without flavopiridol (Flavo; $1 \mu \mathrm{m}$ ) were fixed at the times indicated, immunostained for cyclin D1 $(A)$ or cyclin $\mathrm{E}(B)$, and counterstained with the nuclear dye YOYO-1. The percentage of neurons with intense nuclear cyclin D1 or cyclin E immunoreactivity was determined from three fields of 100 cells from three to four independent experiments. ${ }^{*} p<0.01$ compared with control; ${ }^{*} p<0.05$ compared with control. n.S., Not significantly different compared with lactacystin alone; $c t$, control.

within this time frame all neurons cumulatively show nuclear translocation of cyclins D1 and E. However, we cannot exclude the possibility that this event occurs only in a subpopulation of cortical neurons exposed to lactacystin.

\section{Inhibition of Cdk activity does not prevent ubiquitinated} inclusion formation, but cyclins D1 and E accumulate in such inclusions

We have shown previously that pharmacological inhibition of the proteasome leads not only to neuronal apoptosis but also to formation of cytoplasmic ubiquitinated inclusions in primary cortical neurons (Rideout and Stefanis, 2002) and dopaminergic PC12 cells (Rideout et al., 2001a). Interestingly, such inclusions are only seen in nonapoptotic cells (Rideout et al., 2001a; Rideout and Stefanis, 2002). To determine whether Cdk activity is required for such inclusion formation, we counted the percentage of cells with cytoplasmic ubiquitin-positive inclusions in cultures treated with lactacystin for $36 \mathrm{hr}$ in the presence or absence of flavopiridol. Our previous work has shown that inclusion formation in cortical neurons peaks $\sim 36 \mathrm{hr}$ after lactacystin application, and that inclusions are not detected earlier than $12 \mathrm{hr}$ (Rideout and Stefanis, 2002). As we have seen in our previous work, cytoplasmic ubiquitinated inclusions were detected only in viable neurons on the basis of nuclear morphology. As before, Cdk inhibition prevented the apoptotic death of neurons treated with lactacystin. However, the percentage of neurons with ubiquitinpositive inclusions was unaffected; in fact, the percentage was slightly higher in flavopiridol-treated cultures (Fig. 8A). As a control, cultures exposed to flavopiridol alone were fixed and similarly stained; no inclusions were visible in these cultures (data not shown).

The fact that Cdk activity is not required for inclusion forma- 


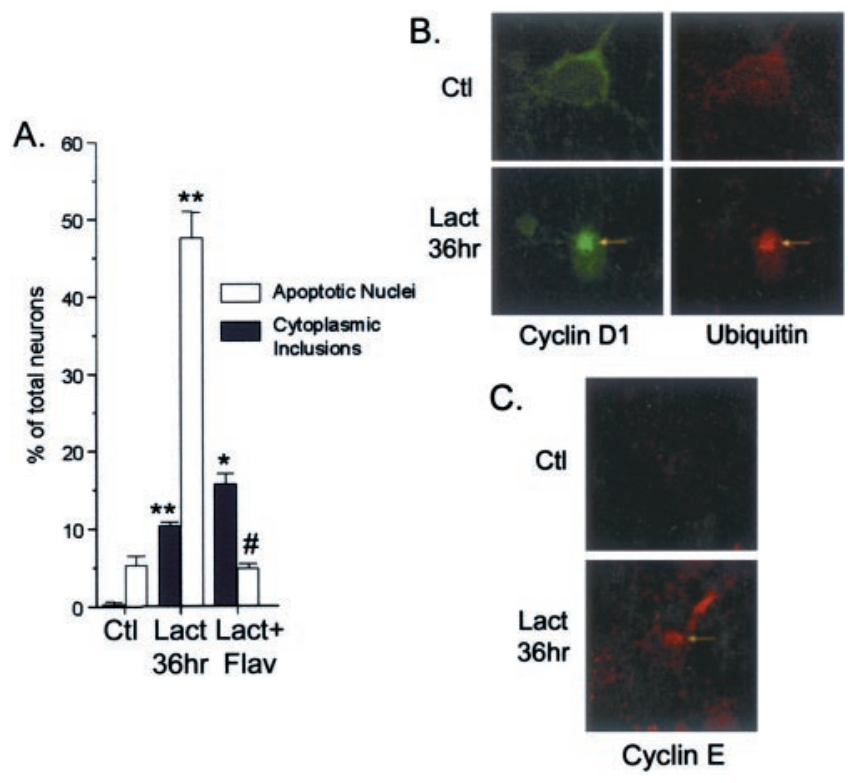

Figure 8. Cyclins D1 and E are sequestered within cytoplasmic inclusions after prolonged inhibition of the proteasome in cortical neurons. A, Neurons were treated with lactacystin (Lact; $10 \mu \mathrm{m}$ ) in the presence or absence of flavopiridol (Flav; $1 \mu \mathrm{m}$ ) for $36 \mathrm{hr}$, fixed, immunostained for anti-ubiquitin, and counterstained with the nuclear dye YOY0-1. The percentage of neurons harboring discrete cytoplasmic ubiquitin-positive inclusions or apoptotic nuclei was determined from three fields of 100 cells from two independent cultures. ${ }^{* *} p<0.001$ compared with control; $" p<0.001$ compared with lactacystin alone; ${ }^{*} p<0.01$ compared with lactacystin alone. $B$, Neurons were treated with lactacystin $(10 \mu \mathrm{m})$ for $36 \mathrm{hr}$, fixed, and immunostained for rabbit anti-ubiquitin (1:100) and mouse anti-cyclin D1 (1:20). A representative confocal image shows colocalization of ubiquitin and cyclin D1 immunoreactivity within a discrete cytoplasmic inclusion (arrow) in lactacystin-treated neurons. C, Neurons were treated as in $B$ and immunostained for rabbit anti-cyclin $E$ (1:100). A representative confocal image shows cyclin E-positive cytoplasmic inclusion (arrow) in a lactacystin-treated neuron. Ctl, Control.

tion in cortical neurons does not exclude the possibility that components of the cell cycle that are normally degraded by the proteasome may accumulate within the cytoplasmic ubiquitinated inclusions at late time points after proteasomal inhibition. To test this possibility, we fixed and immunostained cells cultured for 36 hr with lactacystin, using cyclin D1, cyclin E, and p27 antibodies, in conjunction with ubiquitin antibodies. We did not find any p27 localization within ubiquitinated inclusions (data not shown). In contrast, both cyclin D1 and cyclin E became sequestered within cytoplasmic inclusions in a percentage of neurons 36 hr after lactacystin application (Fig. $8 B, C$ ).

In conjunction, these results indicate that, whereas Cdk activity is not required for inclusion formation in this model, elements of the cell-cycle machinery, such as cyclins D1 and E, which early on translocate to the nucleus, accumulate at later time points after lactacystin application in cytoplasmic inclusions.

\section{Discussion}

Cdk 2, Cdk4, and Cdk6 are required for proteasomal inhibition-induced death of cultured cortical neurons

We show here that aberrant activation of Cdks that function at the $G_{1} / S$ interphase of the cell cycle is required for proteasomal inhibition-induced death of postmitotic cortical neurons. At this stage of the cell cycle, there is activation of the Cdk4/Cdk6/cyclin D1 and Cdk2/cyclin E complexes. The pharmacological Cdki we used in the present work, flavopiridol, inhibits the activities of $\mathrm{Cdk} 2$ and Cdk4 with similar efficiency, while poorly inhibiting the activities of other protein kinases (Losiewicz et al., 1994; Park et al., 1996). Consistent with our pharmacological data, overex-

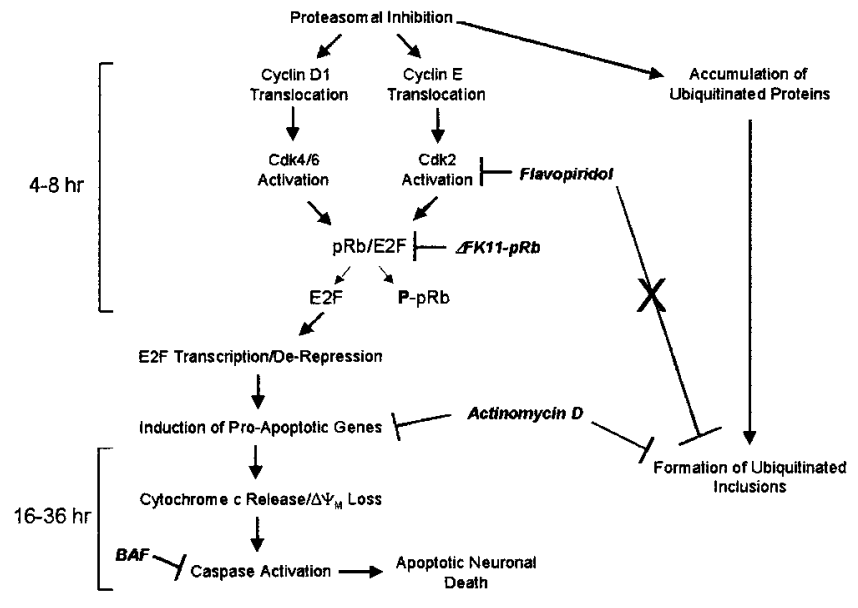

Figure 9. Model of proteasomal inhibitor-induced activation of components of the cell cycle in cultured cortical neurons undergoing apoptosis.

pression of DN forms of Cdk2, Cdk4, or Cdk6, or of the Cdkis p16 or p27, also afforded protection in this model.

Our findings with DN Cdk4 and Cdk6 are similar to those reported for DNA damage- or trophic deprivation-induced apoptosis of cortical and sympathetic neurons (Park et al., 1997, 1998 ) and those with $\beta$-amyloid-induced death of cortical neurons (Giovanni et al., 1999). However, in contrast to our present findings, DN Cdk2 was ineffective in preventing death in those models. However, DN Cdk2 or Cdk3 prevent the tumor necrosis factor- $\alpha$-induced apoptotic death of HeLa cells (Meikrantz and Schlegel, 1996). In addition, in $\mathrm{K}^{+}$-deprivation-induced apoptosis of cultured cerebellar granule neurons, there are alterations in cyclin E immunoreactivity and an early induction of cyclin E-associated activity, implying involvement of the cyclin E/Cdk2 complex in this type of death (Padmanabhan et al., 1999). Together, these data suggest that the particular components of the cell-cycle machinery that are important for neuronal death may depend on the cell death stimulus and/or cell type.

It should be noted that flavopiridol, despite some toxicity, offered prolonged protection against proteasomal inhibitioninduced death. This may be related to the fact that the action of flavopiridol lies upstream of the mitochondrial checkpoint (Figs. 2 and 9). Mitochondrial function is thus preserved in proteasome inhibitor-exposed neurons treated with flavopiridol. This is consistent with results in DNA damage-induced neuronal death (Stefanis et al., 1999; Morris et al., 2001). Cdk inhibition may therefore provide a better therapeutic option compared with caspase inhibition, which does not prevent mitochondrial alterations (present study) (Stefanis et al., 1999; Morris et al., 2001; Rideout and Stefanis, 2001).

\section{Ordering of aberrant cell-cycle activation induced by proteasomal inhibition}

A major substrate for cyclin D1 and E-associated kinase complexes is pRb. To assess this kinase activity, we used an antibody that specifically detects pRb phosphorylated at Ser795, a site targeted by cyclin D1/Cdk4 and cyclin E/Cdk2 (Lundberg and Weinberg, 1998; Harbour et al., 1999). The increase in phosphorylated $\mathrm{pRb}$, beginning at $4 \mathrm{hr}$ after lactacystin exposure, correlated temporally with redistribution of cyclin D1 and cyclin E to the nuclear compartment and the increase in cyclin D1 levels. The fact that nuclear translocation of cyclins D1 and E was not prevented by flavopiridol suggests that it precedes, and leads to, Cdk 
activation (see proposed model in Fig. 9). Interestingly, in the model of $\mathrm{K}^{+}$-deprivation-induced apoptosis of cultured cerebellar granule neurons, Cdk inhibition with flavopiridol prevented cyclin D1 nuclear translocation (Padmanabhan et al., 1999). This again underscores differences among the cell-cycle pathways induced by various cell death stimuli in different neuronal populations.

Consistent with previous results in cerebellar granule neurons (Boutillier et al., 1999), we found an increase in total levels of cyclin D1 by Western immunoblot after inhibition of the proteasome. However, we were unable to detect such an increase for cyclin $\mathrm{E}$. This may be an issue of sensitivity, because at any given point in time a smaller subpopulation of neurons shows cyclin $\mathrm{E}$ nuclear translocation. This is in contrast to our findings with p27, which was not altered after proteasomal inhibition in cortical neurons. In cycling cells, p27 is known to be regulated by the proteasome (Pagano et al., 1995), providing a basis for the phenomenon of cell-cycle arrest after proteasomal inhibition (Fenteany et al., 1995; Pagano et al., 1995; Baldassarre et al., 2000). The apparent absence of p27 regulation in cortical neurons may account for the opposing effects of proteasomal inhibition on cellcycle progression in cycling cells and neurons. This underscores the fact that proteins regulated by the proteasome in nonneuronal, cycling cells are not necessarily regulated by the proteasome in postmitotic neurons.

$\mathrm{pRb}$ is normally bound in a complex with E2F. This complex actively represses the transcription of certain genes. With $\mathrm{pRb}$ phosphorylation, $\mathrm{pRb}$ dissociates from the complex, and $\mathrm{E} 2 \mathrm{~F}$ binds to a new DNA site, where it functions as a direct transcription factor (Harbour and Dean, 2000; Liu and Greene, 2001). The $\Delta$ FK11 mutant form of $\mathrm{pRb}$ that we have used, because of its relative lack of phosphorylation sites, attaches firmly to endogenous E2F, even in the face of Cdk activation. We have shown that expression of $\triangle \mathrm{FK} 11 \mathrm{pRb}$ promotes survival in this model, thus implicating E2F derepression and/or transactivation in this death.

The fact that novel or ongoing transcription is required for death in this model is supported by our present and past results (Rideout and Stefanis, 2002) showing that the transcriptional inhibitor actinomycin D prevents apoptosis and mitochondrial alterations. Actinomycin $\mathrm{D}$, however, does not prevent $\mathrm{pRb}$ phosphorylation (Fig. 3), suggesting that its actions, at least in part, are mediated at the level of E2F derepression and/or transactivation (Fig. 9).

\section{Relationship between inclusion formation, cyclins, Cdk activation, and death}

In addition to cell death, another major phenomenon that occurs after proteasomal inhibition of cortical neurons is the formation of ubiquitinated cytoplasmic inclusions (Rideout and Stefanis, 2002). What is the relationship between cell-cycle components and inclusion formation? As we have reported previously, inclusions begin to appear after $12 \mathrm{hr}$ of lactacystin treatment (Rideout and Stefanis, 2002). Activation of cell-cycle components occurs earlier, and Cdk inhibition does not prevent inclusion formation. However, cyclins D1 and $\mathrm{E}$ also form part of these inclusions. The most likely interpretation of these findings is that aberrant activation of cell-cycle components occurs in a parallel pathway to that of inclusion formation (Fig. 9). The fact that cyclins D1 and $\mathrm{E}$ accumulate in the inclusions is probably a result of their eventual accumulation in the cytoplasm and is unrelated to their role in the initial propagation of the apoptotic pathway.

A related issue is whether the cells that contain nuclear cyclin
D1 and E and those bearing inclusions represent separate subpopulations. If the nuclear translocation of the cyclins is necessary for cell death after inhibition of the proteasome, it is possible that by sequestering these proteins within cytoplasmic ubiquitinated inclusions their deleterious properties are abrogated, giving the cells that harbor such inclusions a survival advantage (Saudou et al., 1998). In this case, neurons that harbor inclusions would be different from those that show nuclear translocation. Alternatively, the same cells that initially show nuclear translocation of cyclins D1 and E may shuttle these proteins to the cytoplasm, where they comprise part of the inclusions. This second possibility is more consistent with the temporal evolution of these phenomena.

In conclusion, our present data indicate that proteasomal inhibition-induced apoptosis of cultured cortical neurons requires aberrant $\mathrm{Cdk}$ activation. The fact that Cdk activation occurred early in the apoptotic pathway, and that Cdk inhibition prevented mitochondrial changes, may have therapeutic implications, especially in diseases such as $\mathrm{AD}, \mathrm{HD}$, or $\mathrm{PD}$, in which proteasomal dysfunction is thought to play a role. The fact that Cdk inhibition does not affect proteasomal inhibition-induced inclusion formation also delineates separate pathways for inclusion formation and death after proteasomal dysfunction.

\section{References}

Baldassarre G, Boccia A, Bruni P, Sandomenico C, Barone MV, Pepe S, Angrisano T, Belletti B, Motti ML, Fusco A, Viglietto G (2000) Retinoic acid induces neuronal differentiation of embryonal carcinoma cells by reducing proteasome-dependent proteolysis of the cyclin-dependent inhibitor p27. Cell Growth Differ 11:517-526.

Boutillier A-L, Kienlen-Campard P, Loeffler J-P (1999) Depolarization regulates cyclin D1 degradation and neuronal apoptosis: a hypothesis about the role of the ubiquitin/proteasome signaling pathway. Eur J Neurosci 11:441-448.

Brown VD, Phillips RA, Gallie BL (1999) Cumulative effect of phosphorylation of pRB on regulation of E2F activity. Mol Cell Biol 19:3246-3256.

Busser J, Geldmacher DS, Herrup K (1998) Ectopic cell cycle proteins predict the sites of neuronal cell death in Alzheimer's disease brain. J Neurosci 18:2801-2807.

Chung KK, Dawson VL, Dawson TM (2001) The role of the ubiquitinproteasomal pathway in Parkinson's disease and other neurodegenerative disorders. Trends Neurosci 24:S7-S14.

Deshmukh M, Johnson Jr EM (1998) Evidence of a novel event during neuronal death: development of competence-to-die in response to cytoplasmic cytochrome $c$. Neuron 21:695-705.

Drexler H (1997) Activation of the cell death program by inhibition of proteasome function. Proc Natl Acad Sci USA 94:855-860.

Fenteany G, Schreiber S (1998) Lactacystin, proteasome function, and cell fate. J Biol Chem 273:8545-8548.

Fenteany G, Standaert RF, Lane WS, Choi S, Corey EJ, Schreiber SL (1995) Inhibition of proteasome activities and subunit-specific amino-terminal threonine modification by lactacystin. Science 268:726-731.

Figueiredo-Pereira M, Berg K, Wilk S (1994) A new inhibitor of the chymotrypsin-like activity of the multicatalytic proteinase complex (20S Proteasome) induces accumulation of ubiquitin-protein conjugates in a neuronal cell. J Neurochem 63:1578-1581.

Giovanni A, Wirtz-Brugger F, Keramaris E, Slack R, Park DS (1999) Involvement of cell cycle elements, cyclin-dependent kinases, $\mathrm{pRb}$, and $\mathrm{E} 2 \mathrm{~F} \times \mathrm{DP}$, in $\beta$-amyloid-induced neuronal death. J Biol Chem 274:19011-19016.

Grunblatt E, Mandel S, Maor G, Youdim MB (2001) Gene expression analysis in $N$-methyl-4-phenyl-1,2,3,6-tetrahydropyridine mice model of Parkinson's disease using cDNA microarray: effect of $R$-apomorphine. J Neurochem 78:1-12.

Harbour JW, Dean DC (2000) The Rb/E2F pathway: expanding roles and emerging paradigms. Genes Dev 14:2393-2409.

Harbour JW, Luo RX, Dei Santi A, Postigo AA, Dean DC (1999) Cdk phosphorylation triggers sequential intramolecular interactions that progressively block Rb functions as cells move through $\mathrm{G}_{1}$. Cell 98:859-869. 
Hsieh FF, Barnett LA, Green WF, Freedman K, Matushansky I, Skoultchi AI, Kelley LL (2000) Cell cycle exit during terminal erythroid differentiation is associated with accumulation of p27(Kip1) and inactivation of cdk2 kinase. Blood 96:2746-2754.

Iwatsubo T, Yamaguchi H, Fujimuro M, Yokosawa H, Ihara Y, Trojanowski JQ, Lee VM (1996) Purification and characterization of Lewy bodies from the brains of patients with diffuse Lewy body disease. Am J Pathol 148:1517-1529.

King RW, Deshaies RJ, Peters JM, Kirschner MW (1998) How proteolysis drives the cell cycle. Science 274:1652-1659.

Lam YA, Pickart CM, Alban A, Landon M, Jamieson C, Ramage R, Mayer RJ, Layfield R (2000) Inhibition of the ubiquitin-proteasome system in Alzheimer's disease. Proc Natl Acad Sci USA 97:9902-9906.

Leroy E, Boyer R, Auburger G, Leube B, Ulm G, Mezey E, Harta G, Brownstein MJ, Jonnalagada S, Chernova T, Dehejia A, Lavedan C, Gasser T, Steinbach PJ, Wilkinson KD, Polymeropoulos MH (1998) The ubiquitin pathway in Parkinson's disease. Nature 395:451-452.

Liu DX, Greene LA (2001) Regulation of neuronal survival and death by E2F-dependent gene repression and derepression. Neuron 32:425-438.

Losiewicz MD, Carlson BA, Kaur G, Sausville EA, Worland PJ (1994) Potent inhibition of CDC2 kinase activity by the flavonoid L86-8275. Biochem Biophys Res Commun 201:589-595.

Lundberg LS, Weinberg RA (1998) Functional inactivation of the retinoblastoma protein requires sequential modification by at least two distinct cyclin-cdk complexes. Mol Cell Biol 18:753-761.

McNaught K, Olanow CW, Halliwell B, Isacson O, Jenner P (2001) Failure of the ubiquitin-proteasome system in Parkinson's disease. Nat Rev Neurosci 2:589-594.

Meikrantz W, Schlegel R (1996) Suppression of apoptosis by dominant negative mutants of cyclin-dependent protein kinases. J Biol Chem 271:10205-10209.

Meng L, Mohan R, Kwok BH, Elofsson M, Sin N, Crews CM (1999) Epoxomicin, a potent and selective proteasome inhibitor, exhibits in vivo antiinflammatory activity. Proc Natl Acad Sci USA 96:10403-10408.

Morris EJ, Keramaris E, Rideout HJ, Slack RS, Dyson NJ, Stefanis L, Park DS (2001) Cyclin-dependent kinases and P53 pathways are activated independently and mediate Bax activation in neurons after DNA damage. J Neurosci 21:5017-5026.

Nagy Z, Esiri MM, Smith AD (1998) The cell division cycle and the pathophysiology of Alzheimer's disease. Neuroscience 87:731-739.

Neame SJ, Rubin LL, Philpott KL (1998) Blocking cytochrome $c$ activity within intact neurons inhibits apoptosis. J Cell Biol 142:1583-1593.

Obin M, Mesco E, Gong X, Haas AL, Joseph J, Taylor A (1999) Neurite outgrowth in PC12 cells. Distinguishing the roles of ubiquitylation and ubiquitin-dependent proteolysis. J Biol Chem 274:11789-11795.

Padmanabhan J, Park DS, Greene LA, Shelanski ML (1999) Role of cell cycle regulatory proteins in cerebellar granule neuron apoptosis. J Neurosci 19:8747-8756.

Pagano M (1997) Cell cycle regulation by the ubiquitin pathway. FASEB J 11:1067-1075.

Pagano M, Tam SW, Theodoras AM, Beer-Romero P, Del Sal G, Chau V, Yew PR, Draetta GF, Rolfe M (1995) Role of the ubiquitin-proteasome pathway in regulating abundance of the cyclin-dependent kinase inhibitor p27. Science 269:682-685.
Park DS, Farinelli SE, Greene LA (1996) Inhibitors of cyclin-dependent kinases promote survival of post-mitotic neuronally differentiated PC12 cells and sympathetic neurons. J Biol Chem 271:8161-8169.

Park DS, Levine B, Ferrari G, Greene LA (1997) Cyclin-dependent kinase inhibitors and dominant negative cyclin-dependent kinase 4 and 6 promote survival of NGF-deprived sympathetic neurons. J Neurosci 17:8975-8983

Park DS, Morris EJ, Padmanabhan J, Shelanski ML, Geller HM, Greene LA (1998) Cyclin-dependent kinases participate in death of neurons evoked by DNA-damaging agents. J Cell Biol 143:457-467.

Park DS, Morris EJ, Bremner R, Keramaris E, Padmanabhan J, Rosenbaum M, Shelanski ML, Geller HM, Greene LA (2000) Involvement of retinoblastoma family members and E2F/DP complexes in the death of neurons evoked by DNA damage. J Neurosci 20:3104-3114.

Qiu JH, Asai A, Chi S, Saito N, Hamada H, Kirino T (2000) Proteasome inhibitors induce cytochrome $c$-caspase-3-like protease-mediated apoptosis in cultured cortical neurons. J Neurosci 20:259-265.

Rideout HJ, Stefanis L (2001) Caspase inhibition: a potential therapeutic strategy in neurological diseases. Histol Histopathol 16:895-908.

Rideout HJ, Stefanis L (2002) Proteasomal inhibition-induced inclusion formation and death in cortical neurons require transcription and ubiquitination. Mol Cell Neurosci 21:223-238.

Rideout HJ, Larsen KE, Sulzer D, Stefanis L (2001a) Proteasomal inhibition leads to formation of ubiquitin/ $\alpha$-synuclein-immunoreactive inclusions in PC12 cells. J Neurochem 78:899-908.

Rideout HJ, Zang E, Yeasmin M, Gordon R, Jabado O, Park DS, Stefanis L (2001b) Inhibitors of trypsin-like serine proteases prevent DNA damageinduced neuronal death by acting upstream of the mitochondrial checkpoint and of p53 induction. Neuroscience 107:339-352.

Sandhu C, Peehl DM, Slingerland J (2000) p16INK4A mediates cyclindependent kinase 4 and 6 inhibition in senescent prostatic epithelial cells Cancer Res 60:2616-2622.

Saudou F, Finbeiner S, Devy D, Greenberg M (1998) Huntingtin acts in the nucleus to induce apoptosis but death does not correlate with the formation of intranuclear inclusions. Cell 95:55-66.

Shimura H, Hattori N, Kubo S, Mizuno Y, Asakawa S, Minoshima S, Shimizu N, Iwai K, Chiba T, Tanaka K, Suzuki T (2001) Familial Parkinson disease gene product, parkin, is a ubiquitin-protein ligase. Nat Genet 25:302-305

Stefanis L, Park DS, Friedman WJ, Greene LA (1999) Caspase-dependent and -independent death of camptothecin-treated embryonic cortical neurons. J Neurosci 19:6235-6247.

Stefanis L, Larsen KE, Rideout HJ, Sulzer D, Greene LA (2001) Expression of A53T mutant but not wild-type $\alpha$-synuclein in PC12 cells induces alterations of the ubiquitin-dependent degradation system, loss of dopamine release, and autophagic cell death. J Neurosci 21:9549-9560.

Tanaka Y, Engelender S, Igarashi S, Rao RK, Wanner T, Tanzi RE, Sawa A Dawson VL, Dawson TM, Ross CA (2001) Inducible expression of mutant $\alpha$-synuclein decreases proteasome activity and increases sensitivity to mitochondria-dependent apoptosis. Hum Mol Genet 10:919-926.

Waelter S, Boeddrich A, Lurz R, Scherzinger E, Lueder G, Lerach H, Wanker EE (2001) Accumulation of mutant huntingtin fragments in aggresome-like inclusion bodies as a result of insufficient protein degradation. Mol Biol Cell 12:1393-1407. 